Check for updates

Cite this: RSC Adv., 2019, 9, 4812

Received 7th December 2018

Accepted 28th January 2019

DOI: 10.1039/c8ra10057h

rsc.li/rsc-advances

\section{Photoswitchable probe with distinctive characteristics for selective fluorescence imaging and long-term tracing $\dagger$}

\begin{abstract}
Zhenzhen Tu, Qian Zhang, Xiuxia Xu, Lianhui Wang, (D) Yan Qian (D) * and Wei Huang*
A photo-switchable and high-contrast bio-imaging indicator 4,4'-(1E,1'E)- $\left(4,4^{\prime}\right.$ - (cyclopentene-1,2diyl)bis(5-methylthiophene-4,2-diyl))bis(methan-1-yl-1-ylidene)bis(azan-1-yl-1-ylidene)bis(2-(benzo [d]thiazol-2-yl)phenol) (BMBT) has been demonstrated, by integrating photochromophore with excited-state intramolecular proton transfer (ESIPT) moiety. The ability of reversible emission switching enables arbitrarily selective labeling or concealing of cells simply by controlling light irradiation. Besides, when the emission was switched on, BMBT is demonstrated to exhibit unique characteristics of aggregation induced emission (AIE), providing a high on-off ratio for favorable bio-imaging. Thus, the non-labeling and easily-controlled selective imaging, as well as good biocompatibility indicates BMBT to be a favorable cell probe with great potentials for functional bioimaging fluorophore.
\end{abstract}

Although there are many conventional fluorescent probes used for fluorescent imaging in the past few years, ${ }^{1-5}$ such as rhodamine, ${ }^{6}$ cyanine dye, ${ }^{7}$ quantum dots, ${ }^{8,9}$ and lanthanide probes, ${ }^{\mathbf{1 0}}$ these fluorescent probes can only respond irreversibly to one event. ${ }^{\mathbf{1 1}, 12}$ In comparison, photochromophores, ${ }^{13}$ which can reversibly response with UV and visible light, ${ }^{\mathbf{1 4}}$ are more valuable fluorescent probes for regional optical marking of interested cells. ${ }^{15,16}$ Because of their favourable characteristics, such as excellent thermal stability, good fatigue resistance and fast response time, diarylethenes derivatives have drawn wide spread concern of researchers. ${ }^{17-20}$ Furthermore, the special optical properties of diarylethenes enable them to be suitable for long time and real time monitoring in bioimaging.

The significance of targeted imaging of fluorescence probe for bio-samples in vitro or in vivo is well-known for their high sensitivity, non-invasiveness, real-time detection and especially selectivity. ${ }^{21-24}$ However, the synthesis of these targeting materials is usually complicated. Moreover, for the same type of cells, no selectivity is demonstrated by these complex targeting agents. $^{21-24}$ Therefore, easy-prepared and easy-controlled nonlabeling fluorescence imaging agents have always been pursued by both the industry and scientific communities. Among the above-mentioned photochromophores, diarylethenes are expected to be promising photochromic imaging

Key Laboratory for Organic Electronics \& Information Displays, Institute of Advanced Materials (IAM), Nanjing University of Posts \& Telecommunications, 9 Wenyuan Road, Nanjing, 210023, China. E-mail: iamyqian@njupt.edu.cn; iamwhuang@njupt.edu.cn; Fax: +86258586 6999; Tel: +862585866008

$\dagger$ Electronic supplementary information (ESI) available: Procedures and results of additional experiments See DOI: 10.1039/c8ra10057h candidates due to their favorable characteristics described in preceding paragraph. Prospectively, they can be used for selective long-term tracing if the photostability can be ensured.

In our previous work, a series of AIE-active excited-state intramolecular proton transfer (ESIPT) complexes have been demonstrated to be good bio-imaging candidate with many advantages such as simple preparation, good biocompatibility, high quantum yields, fast cell staining as well as long-term antiphotobleaching. ${ }^{25-27}$ Sometimes, ESIPT compounds exhibit dual emission, originated from keto and enol state, respectively. This caused extremely fast four-level photophysical cycle $\left(\mathrm{E}-\mathrm{E}^{*}-\mathrm{K}^{*}-\right.$ $\mathrm{K}-\mathrm{E}$ ), mediated by intramolecular $\mathrm{H}$-bonds immediately after photoexcitation, enables two emissions. ${ }^{28,29}$ Herein, we have demonstrated a new type of multifunctional bio-imaging materials based on facile synthesis design concept, by introducing a photochromic diarylethene moiety to enable regional emission turn-on, and introducing an ESIPT moiety to allow good photo stability for long-term tracing. To the best of our knowledge, this is the first example with integrating abilities of non-labeling selectively long-term regional tracing.

The new diarylethene derivative (BMBT, see the molecular structure in Scheme 1) has been synthesized (Fig. S1†) via condensation reaction of 5-amino-2-(benzo[d]thiazol-2-yl) phenol with 4,4-(cyclopentene-1,2-yl)-bis(5-methyl-thiophene-

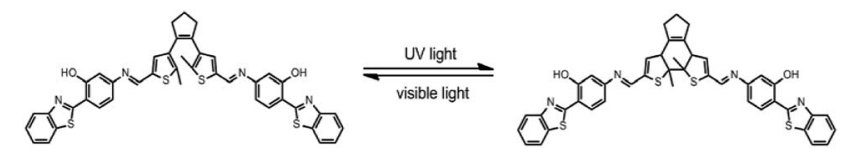

Scheme 1 Structure and photochromic process of BMBT. 
2-formaldehyde), according to a previous reported procedure. ${ }^{\mathbf{3 0 , 3 1}}$ The molecular structures and purities were confirmed by ${ }^{1} \mathrm{H}$ NMR spectroscopy and mass spectroscopy (Fig. S6 and $\mathrm{S} 7, \dagger$ see the synthesis details and full molecular characterizations in the ESI†).

The photoirradiation-induced changes in absorption and fluorescence spectra at room temperature are investigated in THF/DMEM mixture $\left(1.0 \times 10^{-5} \mathrm{~mol} \mathrm{~L}^{-1}\right)$. The open-ring isomer mainly exhibits two absorption peaked at 304 and $380 \mathrm{~nm}$, respectively (Fig. S2 $\dagger$ ). This is ascribed to the internal charge transfer and $\pi-\pi$ transition of 2 -(2'-hydroxy-phenyl) benzothiazole (HBT), ${ }^{\mathbf{1 0}, 30}$ coupled with the CT inside the HBT unit from the hydroxyphenyl ring to the benzothiazole ring (Scheme 1). BMBT exhibits two emissions; one blue emission peak around $458 \mathrm{~nm}$ and a red emission peak around $600 \mathrm{~nm}$, corresponding to the enol and keto emission, respectively (Fig. 1). Upon irradiation with ultraviolet light at $365 \mathrm{~nm}$, the absorption band at longer wavelength region centered at $595 \mathrm{~nm}$ increased obviously with irradiation time (Fig. S2 $\dagger$ ). This is caused by the formation of the closed-ring isomer (see the photochromic reaction in Scheme 1). Correspondingly, due to spectroscopic overlap between this longer-wavelength absorbance with the red emission ranged from 560-700 nm, the relative intensity of the red emission substantially decreases with the UV irradiation (Fig. 1), because of efficient energy transfer. Upon further visible light $(\lambda=520 \mathrm{~nm})$ irradiation, the closed-ring isomer transfers back to the initial open-ring isomer, and thus the longer-wavelength absorption decreases and the red emission restores. This indicates good reversibility of the photochromic reaction.

Excitingly, BMBT exhibit prominent characteristics of Aggregation-Induced Emission (AIE). ${ }^{31-33}$ The fluorescence intensity of BMBT in THF solution was relatively weak, while the powder or nanoparticles of the material dispersed in DMEM buffer exhibited a significantly enhanced emission (Fig. 2). When the DMEM buffer fraction was increased gradually from $0 \%$ to $20 \%$, the fluorescence intensity only slightly enhanced. When the DMEM fraction was further increased to $40 \%$, the

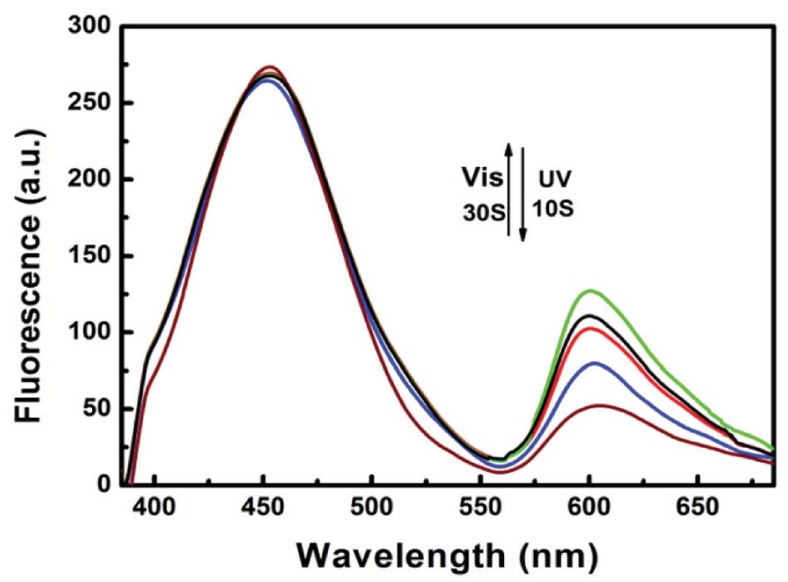

Fig. 1 Fluorescence emission changes of BMBT in THF/DMEM (1: 200, vol : vol) mixture upon irradiation with $365 \mathrm{~nm}$ light $\left(\lambda_{\mathrm{ex}}=385\right.$ $\mathrm{nm})$ and visible light $\left(\lambda_{\mathrm{ex}}=520 \mathrm{~nm}\right)$.



Fig. 2 Fluorescence spectra of $1 \times 10^{-5} \mathrm{M}$ BMBT in the THF/DMEM mixture at different water fractions $\left(\lambda_{\mathrm{ex}}=385 \mathrm{~nm}\right)$.

emission exhibits a significant enhancement. The total intensity at the blue enol emission increased more than 10 times at $100 \%$ DMEM fraction as compared with that in THF solution. This AIE property enables high signal-to-noise ratios for favorable bioimaging (Fig. S3†).

Based on the good reversibility of light response and AIE characteristics, the practical application of the BMBT as bioprobe was further investigated. The biological imaging of BMBT was observed by using confocal laser scanning microscopy (CLSM). Blue luminescence in the cytoplasm of HeLa cells was observed after incubation with a THF/DMEM ( $1: 200$, vol : vol) solution of BMBT $(20 \mu \mathrm{M})$ for $30 \mathrm{~min}$ at $37^{\circ} \mathrm{C}$ (Fig. 2 inset). The overlay of luminescent images and bright-field images confirmed that BMBT was located mainly in the cytoplasm of cells rather than the membrane and nucleus (Fig. S3 $\dagger$ ). Intense intracellular luminescence with a high signal-to-noise ratio $\left(I_{1}\right)$ $I_{2}>7$ ) was detected between the cytoplasm (regions 3 and 1) and nucleus (region 2), also implying weak even few nuclear uptake of BMBT (Fig. S4 $\dagger$ ). Besides, BMBT has a low cytotoxicity with the cellular viabilities estimated to be greater than $85 \%$ after $24 \mathrm{~h}$ incubation with the highest cultural concentration of 50 $\mu \mathrm{M}$ BMBT (Fig. S5 $\dagger$ ).

The luminescence switching of BMBT can also be achieved while alternating UV and visible light illumination in fixed HeLa cells. Cells (shown in red circle, Fig. 3a) were irradiated with $488 \mathrm{~nm}$ light $(0.5 \mathrm{~mW})$ for $3 \mathrm{~min}$, the blue fluorescence of the irradiated cells was lamped off while the surrounding cells remained almost unchanged. Such fluorescence quenching is likely ascribed to the intramolecular fluorescence resonance energy transfer of BMBT, due to the intensified shortwavelength absorption band (270-450 nm) of the closed-open form of BMBT with the blue emission band (420-560 nm). ${ }^{34}$ Upon irradiation with $405 \mathrm{~nm}$ light $(1.25 \mathrm{~mW})$ the fluorescence of all the selected cell was rapidly recovered within $1 \mathrm{~min}$, caused by decrease of the relative intensity of the $270-450 \mathrm{~nm}$ absorption. The fluorescence can be repeatedly erased and recovered many rounds without significant fluorescence quenching, which was hardly achieved by conventional fluorophores (Fig. 3b). ${ }^{35,36}$ 
a
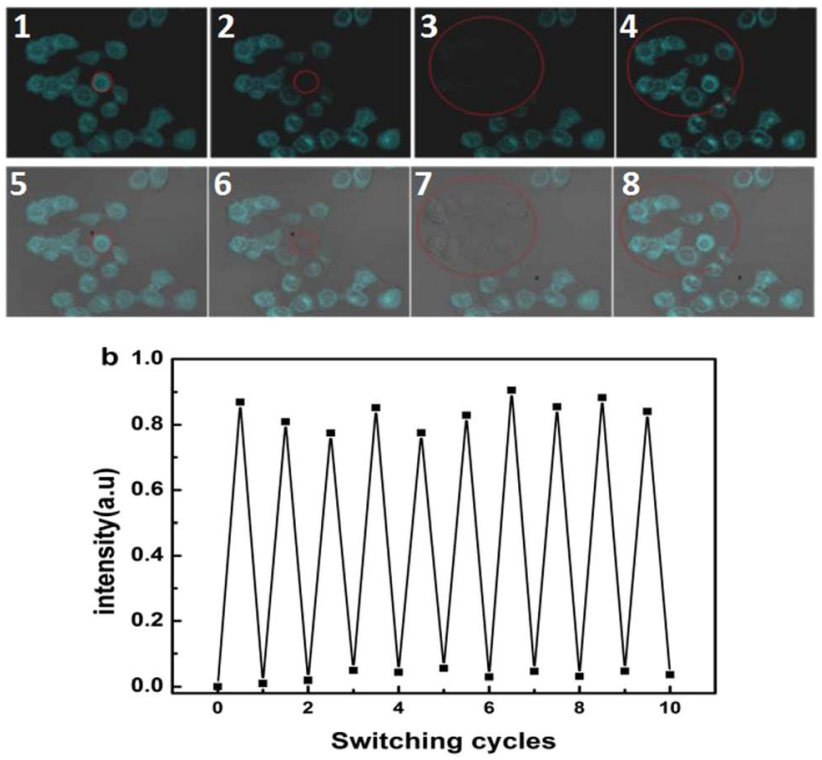

Fig. 3 (a) CLSM image (above) and the overlay image (bottom) of fixed HeLa cells incubated with $20 \mu \mathrm{M} \mathrm{BMBT}$ for $30 \mathrm{~min}$ at $37^{\circ} \mathrm{C}(1)$ and (5) in original state; (2) and (6) irradiated by $488 \mathrm{~nm}$ light $(0.5 \mathrm{~mW})$ for a single cell; (3) and (7) all cells, and; (4) and (8) recovered by $405 \mathrm{~nm}$ light (1.25 $\mathrm{mW}$ ). (b) Fluorescence switching of fixed HeLa cells by alternating UV ( $405 \mathrm{~nm}, 1.25 \mathrm{~mW}, 10 \mathrm{~s} /$ time) and visible ( $488 \mathrm{~nm}, 0.5 \mathrm{~mW}, 3 \mathrm{~min} / \mathrm{time})$ light illumination ( $\lambda_{\mathrm{ex}}=405 \mathrm{~nm}$ ).

This characteristic of selectively opto-marking or de-marking of cells may be used for non-invasive and dynamic tracing of the interested objects in vitro. That is, BMBT can arbitrarily optolabel or de-label interested cells without affecting cell proliferation. As shown in Fig. 4, the cell marked in the red circle was treated with visible light illumination ( $488 \mathrm{~nm}, 0.5 \mathrm{~mW}, 3 \mathrm{~min}$ ), and its fluorescence was effectively quenched. Cells division of the remaining "bright" cells was observed under the microscope field of vision for a long-term tracing with time up to $36 \mathrm{~h}$. Multiple new cells were produced, as indicated by upper white arrow. Even, as indicated by bottom white arrow, the tacked cells were observed to be doubled. The high brightness of the fluorescence in the proliferated cells indicated the good photo stability of BMBT. This indicated that BMBT can be used as
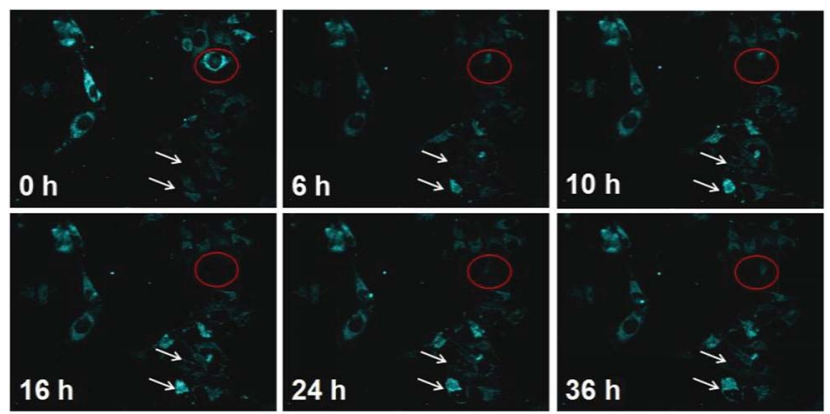

Fig. 4 Cells was treated with visible $(488 \mathrm{~nm}, 0.5 \mathrm{~mW}, 3 \mathrm{~min})$ light illumination for erasing the fluorescence. The remaining bright cells were incubated for another 36 hours, which were observed to be amplified normally $\left(\lambda_{\mathrm{ex}}=405 \mathrm{~nm}\right)$. a cell marker for arbitrarily selective erasing the fluorescence of the designated cell. It can also be used for selective lighting their emission by making them as the remaining bright cells after selective photo-erasing or selective photo-recovering after full erasing. This long-term tracking with non-labeling and selective optically marking or de-marking is seldom reported by other photochromophore-based bioimaging agents. ${ }^{37}$ Herein, the excellent anti-photo bleaching characteristic in the longterm tracing is attributed to high photo stability of the HBT moiety. ${ }^{26,27}$

\section{Conclusions}

To summarize, a photochromic diarylethene based on 2-(2'hydroxyphenyl)benzothiazole called BMBT was designed and investigated to be an luminescence probe, which has an outstanding switchable photochromic characteristic for arbitrarily optically labeling or delabeling interested cells without affecting cell proliferation. As far as we know, this is the first bioimaging fluorophore that simultaneously exhibit unique characteristics of aggregation induced emission (AIE) and nonlabeling selectively imaging for biological detection in vitro. Further investigation for the in vivo application is undergoing.

\section{Conflicts of interest}

There are no conflicts to declare.

\section{Acknowledgements}

This study was funded by the National Natural Science Foundation of China $(61605085,21373114,21573111,21603106)$, National Key Basic Research Program of China (973) (2012CB933301), Project funded by the Priority Academic Program Development of Jiangsu Higher Education Institutions, PAPD (YX030002), Program for Changjiang Scholars and Innovative Research Team in University (IRT1148), Research Foundation from Nanjing University of Posts and Telecommunications (XK0300915081), Qing Lan Project of Jiangsu Province and NUPT 1311 Project, Jiangsu Key Laboratory for Biosensors, Synergetic Innovation Center for Organic Electronics and Information Displays, Jiangsu National Synergetic Innovation Center for Advanced Materials (SICAM).

\section{Notes and references}

1 Z. Chen, K. Y. Zhang, X. Tong, Y. Liu, C. Hu, S. Liu, Q. Yu, Q. Zhao and W. Huang, Adv. Funct. Mater., 2016, 26, 43864396.

2 Y. Zhang, S. Li and Z. Zhao, Anal. Chem., 2016, 88, 12380-12385. 3 Y. Zhang, T. T. Shen, A. M. Kirillov, W. S. Liu and Y. Tang, Chem. Commun., 2016, 52, 7939-7942.

4 J. Huang, L. Ying, X. Yang, Y. Yang, K. Quan, H. Wang, N. Xie, M. Ou, Q. Zhou and K. Wang, Anal. Chem., 2015, 87, 87248731.

5 J. Shangguan, D. He, X. He, K. Wang, F. Xu, J. Liu, J. Tang, X. Yang and J. Huang, Anal. Chem., 2016, 88, 7837-7843. 
6 A. Sahana, A. Banerjee, S. Lohar, B. Sarkar, S. K. Mukhopadhyay and D. Das, Inorg. Chem., 2013, 52, 3627-3633.

7 J. R. Carreon, K. M. Stewart, K. P. Mahon, S. Shin and S. O. Kelley, Bioorg. Med. Chem. Lett., 2007, 17, 5182-5185.

8 Y. C. Lu, J. Chen, A. J. Wang, N. Bao, J. J. Feng, W. P. Wang and L. X. Shao, J. Mater. Chem., 2015, 3, 73-78.

9 X. D. Zhang, H. X. Wang, H. Wang, Q. Zhang, J. F. Xie, Y. P. Tian, J. Wang and Y. Xie, Adv. Mater., 2014, 26, 44384443.

10 J. C. G. Bunzli, Lanthanide Luminescence for Biomedical Analyses and Imaging, Chem. Rev., 2010, 110, 2729-2755.

11 H. Takakusa, K. Kikuchi, Y. Urano, S. Sakamoto, K. Yamaguchi and T. Nagano, J. Am. Chem. Soc., 2002, 124, 1653-1657.

12 M. Zhang, M. X. Yu, F. Y. Li, M. W. Zhu, M. Y. Li, Y. H. Gao, L. Li, Z. Q. Liu, J. P. Zhang and D. Q. Zhang, J. Am. Chem. Soc., 2007, 129, 10322-10323.

13 W. L. Li, C. H. Jiao, X. Li, Y. S. Xie, K. Nakatani, H. Tian and W. H. Zhu, Angew. Chem., Int. Ed., 2014, 53, 4603-4607.

14 M. Q. Zhu, L. Y. Zhu, J. J. Han, W. W. Wu, J. K. Hurst and A. D. Q. Li, J. Am. Chem. Soc., 2006, 128(13), 4303-4309.

15 L. Y. Zhu, W. W. Wu, M. Q. Zhu, J. J. Han, J. K. Hurst and A. D. Q. Li, J. Am. Chem. Soc., 2007, 129, 3524-3526.

16 J. Wiedenmann, S. Ivanchenko, F. Oswald, F. Schmitt, C. Rocker, A. Salih, K. D. Spindler, G. U. Nienhaus and F. P. Eos, Proc. Natl. Acad. Sci. U. S. A., 2004, 101, 1590515910.

17 H. Tian, B. Qin, R. X. Yao, X. L. Zhao and S. J. Yang, Adv. Mater., 2003, 15, 2104-2107.

18 M. Irie, T. Fulcaminato, K. Matsuda and S. Kobatake, Chem. Rev., 2014, 114, 12174-12277.

19 C. Yun, J. You, J. Kim, J. Huh and E. Kim, J. Photochem. Photobiol., C, 2009, 10, 111-129.

20 G. L. Lv, B. P. Cui, H. C. Lan, Y. Wen, A. Y. Sun and T. Yi, Chem. Commun., 2015, 51, 125-128.

21 W. Wang, Y. Huang, S. Wang, Y. Zhou, W. Huang, Y. Feng, W. Zhang, W. Yu, Q. Zhou, M. Chen and M. Fang, Spectrochim. Acta, Part A, 2017, 187, 110-118.
22 W. Xu, P. Ma, Q. Diao, L. Xu, X. Liu, Y. Sun, X. Wang and D. Song, Sens. Actuators, B, 2017, 252, 86-94.

23 W. Xu, G. Han, P. Ma, Q. Diao, L. Xu, X. Liu, Y. Sun, X. Wang and D. Song, Sens. Actuators, B, 2017, 251, 366-373.

24 S. Du, D. Wang, J. S. Lee, B. Peng, J. Ge and S. Q. Yao, Chem. Commun., 2017, 53, 8443-8446.

25 Y. Qian, M. M. Cai, L. H. Xie, G. Q. Yang, S.-K. Wu and W. Huang, ChemPhysChem, 2011, 12, 397-404.

26 Z. Tu, M. Liu, Y. Qian, G. Yang, M. Cai, L. Wang and W. Huang, RSC Adv., 2015, 5, 7789-7793.

27 Z. Tu, Q. Zhang, M. Liu, Y. Qian, L. Wang and W. Huang, J. Mater. Sci., 2016, 51, 2972-2979.

28 Y. Qian, S. Li, G. Zhang, Q. Wang, S. Wang, H. Xu, C. Li, Y. Li and G. Yang, J. Phys. Chem. B, 2007, 111, 5861-5868.

29 M. Cai, Z. Gao, X. Zhou, X. Wang, S. Chen, Y. Zhao, Y. Qian, N. Shi, B. Mi, L. Xie and W. Huang, Phys. Chem. Chem. Phys., 2012, 14, 5289-5296.

30 Y. Cheng, C. Sun, X. Ou, B. Liu, X. Lou and F. Xia, Chem. Sci., 2017, 8, 4571-4578.

31 Q. Y. Fang, J. W. Li, S. Y. Li, R. H. Duan, S. Q. Wang, Y. P. Yi, X. D. Guo, Y. Qian, W. Huang and G. Q. Yang, Chem. Commun., 2017, 53, 5702-5705.

32 H. Y. Li, Z. G. Chi, B. J. Xu, X. Q. Zhang, Z. Y. Yang, X. F. Li, S. W. Liu, Y. Zhang and J. R. Xu, J. Mater. Chem., 2010, 20, 6103-6110.

33 S. Dong, Z. Li and J. Qin, J. Phys. Chem. B, 2009, 113, 434-441. 34 G. Y. Jiang, S. Wang, W. F. Yuan, L. Jiang, Y. L. Song, H. Tian and D. B. Zhu, Chem. Mater., 2006, 18, 235-237.

35 M. Heilemann, S. van de Linde, M. Schuttpelz, R. Kasper, B. Seefeldt, A. Mukherjee, P. Tinnefeld and M. Sauer, Angew. Chem., Int. Ed., 2008, 47, 6172-6176.

36 A. Negre-Salvayre, N. Auge, C. Duval, F. Robbesyn, J. C. Thiers, D. Nazzal, H. Benoist and R. Salvayre, Methods Enzymol., 2002, 352, 62-71.

37 Y. Zou, T. Yi, S. Xiao, F. Li, C. Li, X. Gao, J. Wu, M. Yu and C. Huang, J. Am. Chem. Soc., 2008, 130, 15750-15751. 